\title{
Diagnosis and Management of Cryptogenic Multifocal Ulcerative Stenotic Enteritis with Nephrotic Syndrome: A Systematic Review
}

\author{
Z. W. LIU, B. GAO, YI WANG, JIA QI LIU AND BAI YE*
}

Department of Gastroenterology, Jiangsu Province Hospital of Chinese Medicine, Nanjing, Jiangsu 210029, China

Liu et al.: Cryptogenic Multifocal Ulcerative Stenotic Enteritis with Nephrotic Syndrome

\begin{abstract}
Chronic or recurring subileus condition arising from several tiny intestinal fibrous strictures and several shallow ulcers of the small intestines characterizes cryptogenic multifocal ulcerative stenotic enteritis, an exceedingly uncommon disorder characterized by chronic or relapsing enteritis (asymptomatic at times). It is uncertain as the cause and the pathology is not fully understood. Glucocorticosteroids are recommended for long-term therapy. However, most individuals end up becoming dependent on corticosteroids by increasing the ability to conduct accurate diagnostic work and the capacity to do an endoscopic therapy for an obstruction, deep enteroscopy may eliminate the necessity for surgery and save patients excessive small bowel resections. Whether the onset of cryptogenic multifocal ulcerative stenotic enteritis in this patient is related to thrombotic microangiopathy is still unclear and it is more likely to consider secondary changes in this disease. Although it's an uncommon disorder, we recommend that cryptogenic multifocal ulcerative stenotic enteritis (chronic or relapsing subileus episodes resulting from numerous small intestinal strictures) should be taken into consideration when chronic or recurrent subileus episodes develop from several shallow ulcers of the small intestines. Endoscopic therapy of stenosis, if effective, can avoid surgery and unnecessary small bowel resections.
\end{abstract}

Key words: Cryptogenic multifocal ulcerative stenotic enteritis, glucocorticosteroids, intestinal fibrous strictures, enteroscopy

Chronic or recurring subileus condition arising from several tiny intestinal fibrous strictures and several shallow ulcers of the small intestines characterizes cryptogenic multifocal ulcerative stenotic enteritis (CMUSE), an exceedingly uncommon disorder characterized by chronic or relapsing enteritis (asymptomatic at times) ${ }^{[1]}$. It is uncertain as to the cause and the pathology is not fully understood. Glucocorticosteroids are recommended for long-term therapy. However, most individuals end up becoming dependent on corticosteroids by increasing the ability to conduct accurate diagnostic work and the capacity to do an endoscopic therapy for an obstruction, deep enteroscopy may eliminate the necessity for surgery and save patients from excessive small bowel resections ${ }^{[2]}$.

\section{HISTORY OF THE DISEASE}

After the late 50s and early 60s, more case reports and short series appeared. Okabe and Sakimura originally reported the first Japanese instances of CMUSE in
1968 in a study done by Matsumoto et al..$^{[2]}$. Chronic nonspecific multiple ulcers of the small intestine (CNSU) is the name given to this condition in Japan, where Japanese gastroenterologists have referred to it as $\mathrm{CNSU}^{[2]}$.

All of these early reports are difficult to analyze now since the ability to identify specific diseases was restricted in those days. Our ability to explore the whole small intestine (all 18-21 cm) by endoscopy and to acquire several biopsy specimens, together with use of modern diagnostic technologies like flow cytometry and/or immunohistochemistry, means it is now able to perform a deep examination on the small intestine. Because of this, different entities can be re-discovered ${ }^{[3]}$.

\section{EPIDEMIOLOGY}

CMUSE is an uncommon condition. This entity has been probably greatly underdiagnosed or misdiagnosed,

*Address for correspondence

E-mail: yebaiszyy@163.com 
especially with Crohn's disease and non-steroidal antiinflammatory drugs (NSAIDs) induced enteropathy ${ }^{[3]}$. CMUSE was responsible for 12 of the occurrences of couverture maladie universelle (CMU) hospitalization that occurred in France. This study is based on research that began by analyzing the medical data of 220 French gastrointestinal clinics. In a study published in the journal of Ophthalmology, one of the research conducted in 2012 presented a collection of 15 instances from Japan that included a detailed evaluation of the literature. The study found that CMUSE (diagnosed between 1994 and 2009 at a single tertiary care centre) occurred in three instances in the sample ${ }^{[3]}$. A study conducted by Chinese author Chang et al. ${ }^{[4]}$ studied CMUSE in individuals that were diagnosed by methods of twin balloon enteroscopy (a series from 6 university hospitals in Korea).

\section{CAUSES AND CONSEQUENCES}

CMUSE has not yet been identified as the cause and the mechanism of causation is also not completely understood. Some writers believe that this creature does not exist and that everything is, in fact, a mass hallucination.

Systemic glucocorticosteroids, such as cortisone, favour CMUSE immunological pathogenesis. Approximately $60-90 \%$ of these individuals become corticodependent over a period of up to 3-24 or even $25 \mathrm{~d}$. This might be the solution to aetiology and pathophysiology, because fibrous tissue might be overstimulated ${ }^{[5,6]}$. CMUSE is often linked to chronic subileus episodes since this is one of the primary CMUSE indicators. A third growth factor, transforming growth factor beta (TGF- $\beta$ ), has also been shown to stimulate fibroblast proliferation. This process may be aided by the following growth factors: Interleukin-6 (IL-6), interleukin-8 (IL-8), tumour necrosis factor alpha $(\mathrm{TNF}-\alpha)$, fibroblast growth factors (FGF-2), granulocyte-macrophage colonystimulating factor (GM-CSF) and TGF- $\beta$. In addition to the aforementioned fibroblast and endothelium-derived growth factors, other similar factors are released by fibroblasts and endothelial cells, such as the connective tissue growth factor (CTGF). Collagen degradation by the family of matrix metalloproteinases (MMPs) known as collagenases begins with the breakdown of collagen by a different family of MMPs called collagenases. The aforementioned protease-secreting matrix cells, neutrophils and macrophages are all active. In persons who are in good condition, collagen breakdown can occur rapidly and begins the moment collagen is made.
We believe that multifocal small intestine collagen degradation might be a significant pathogenetic factor in CMUSE. Fibrous tissue inflammatory reaction occurs in $\mathrm{CMUSE}^{[7-9]}$.

The suggested name CMUSE can be described as an "atypical vasculitis". On the other hand, however, any kind of vasculitis of any source, no matter what, should be classified just as "vasculitis." Amino acid derivative of chitin, chitosan and chitosan polysulfate are frequently discovered in the involvement of the small intestine in Churg-Strauss syndrome, systemic lupus erythematosus, Buerger's disease, Wegener's granulomatosis, Weber-Christian disease and systemic sclerosis. Very infrequently, vasculitis is linked with multifocal stenosis of the small intestine, including perforation. Because of this, vasculitis tends to have a systemic component (kidneys, skin, joints, lungs, etc.) [10,11]. Extraintestinal involvement was identified in none of our three patients and histopathology detected no evidence of vasculitis in the small intestine. One of the authors was unable to find arteritis in his cohort. The hypothesis that CMUSE may be linked to C2 complement deficit was first proposed by one author, but was not verified by other researchers. In a study done by Fraile et al. it was discovered that X-linked recessive reticulate pigmentary condition is connected to CMUSE[12].

A major clinical symptom is the presence of several tiny intestinal strictures. Anaemia related to minor intestinal occult blood loss is typically reported in patients with chronic iron insufficiency. Patients are commonly found to have weariness, edema and growth retardation and these problems generally recur as a result of their iron deficiency. However, diarrhoea, malabsorption, hematochezia and fever are infrequently seen in conjunction with these conditions. It is possible for anaemia to be present before a diagnosis of CMUSE.

Several surgeries were required since many patients developed symptomatic strictures from CMUSE and at least one of the strictures needed to be resected. The clinicopathological characteristics of CMUSE have been compiled as despite the surgeries, 7 out of the 10 patients had symptoms in return and 4 individuals experienced strictures reoccurrence. Neutrophils and eosinophils formed the inflammatory infiltration. However, administration of glucocorticosteroids resulted in steroid dependency. A group of researchers obtained data on 2 CMUSE instances found by methods of twin balloon enteroscopy in 48 individuals (a series 
from 6 university hospitals in Korea). Both people experienced recurring stomach discomfort that was persistent and long-term. Patient A was diagnosed with recurrent melena for $41 \mathrm{mo}$, while the other patient was presented for a capsule endoscopic surgery due of a retained endoscope. No indication of arteritis was observed on mesenteric arteriography. CMUSE has mixed inflammatory infiltration (neutrophils, monocytes and eosinophils), tiny intestinal stenosis and shallow ulcers ${ }^{[13,14]}$.

Intestinal strictures, which have previously only been seen in adults, have been discovered in adolescents and middle-agers. Additionally, superficial ulceration of the mucosa and submucosa has been observed, as well as a chronic or relapsing clinical course (even after surgery). On top of that, no biological signs of systemic inflammatory reaction are found and glucocorticosteroids prove to be beneficial.

CMUSE hospitalized 12 patients between 1965 and 1993 was reported by Perlemuter et al. ${ }^{[15]}$. Although all 10 patients had surgery, 7 experienced recurrence of symptoms and 4 reported stricture recurrence. Neutrophils and eosinophils were found within an inflammatory infiltrate, which was comprised of them. The use of glucocorticosteroids was successful, but required the development of steroid dependency. A group of researchers including Chang, et al. obtained data on 2 CMUSE instances found by methods of twin balloon enteroscopy in 48 individuals (a series from 6 university hospitals in Korea). Both patients complained from persistent abdominal discomfort that came and went. Recurrent melena (seen for $41 \mathrm{mo}$ ) was seen in one patient, while a case of retained capsule endoscope was seen in another. In a mesenteric arteriogram, there was no sign of arteritis (inflammation of the small arteries inside the abdomen).

\section{DIAGNOSIS}

In the event of CMUSE, diagnosis should start with reviewing a patient's medical history, observing his or her symptoms, having an abdominal computed tomography (CT) or magnetic resonance imaging (MRI), doing enteroclysis and analyzing the histology of the small intestine. Generally in chest anteroposterior radiograph: no obvious abnormality was found.

We can find the same part in MRI and Copper Dopper. MRI of small intestine: Segmental thickening of part of ileum wall, considering inflammatory bowel disease suspicious, please combine with clinical; the mesenteric lymph nodes in the middle abdomen were slightly enlarged, as shown in fig. 1.

Special Issue 5, 2021

Indian Journal of Pharmaceutical Sciences 


\section{Microscopic diagnosis:}

Multiple ileal ulcers with stenosis (CMUSE); colonic polyps was found.

\section{Pathology:}

The pathology of oral enteroscopy showed chronic active enteritis (proximal ileum) with hyperplasia, dilation and hyperemia of lamina propria. No positive bacteria were found by special staining. Special staining results: acid fast (-), silver staining (-), periodic acidSchiff (PAS) (-); Special staining: Masson's trichrome staining showed hyperplasia of collagen fiber tissue, Van Gieson's (VG) stain showed hyperplasia of collagen fiber tissue, as shown in fig. 2. The pathology of anal enteroscopy showed chronic active enteritis (distal ileum) with ulceration, as shown in fig. 3. Immunofluorescence examination of renal tissue (half a year ago, outer court): (Glomeruli) there were 8 glomeruli, 1/8 of which were sclerotic spheres and immunoglobulin $\mathrm{M}\left(\mathrm{IgM}^{+}\right)$and complement $3\left(\mathrm{C}^{++}\right)$ were diffusely distributed and deposited in mesangial area and vascular loop. Immunoglobulin $\mathrm{G}$ ( $\mathrm{IgG}$ ), immunoglobulin A (IgA), complement component 1q $(\mathrm{C} 1 \mathrm{q}), \kappa$ light chain and $\lambda$ light chain were all negative; (renal tubule) there was no deposition of immune complex and complement in the basement membrane of renal tubule; (vascular wall) there was no immune complex and complement deposition in the blood vessels of the glottic region, the capillary between the tubes and the interstitial vessels (tube type) negative.

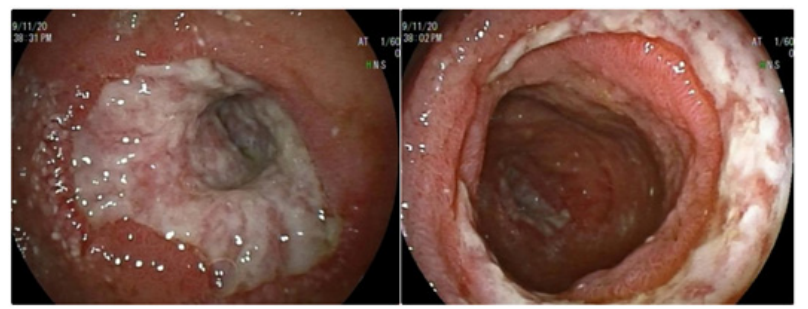

Fig. 2: Superficial annular ulcer was found by oral enteroscopy

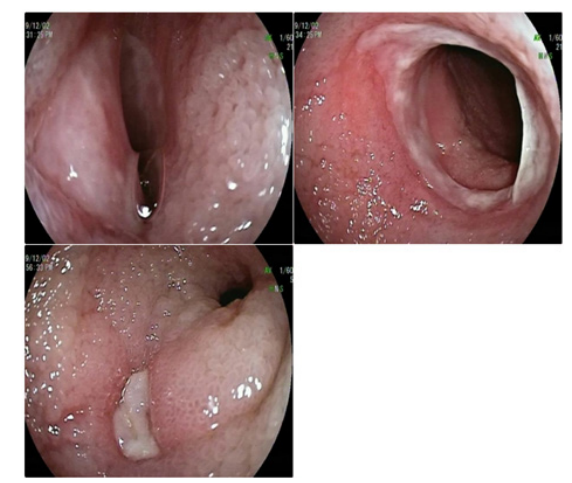

Fig. 3: Through anorectal enteroscopy, superficial annular ulcer was found and a non-circumferential ulcer was also seen
The diagnosis ofCMUSE is not confirmed by any specific laboratory testing. We have one instance of CMUSE with a retained capsule, as well as one additional article reporting on the issue. We documented the spontaneous dissolution of a preserved video capsule before. Most importantly, Crohn's disease must be eliminated in the differential diagnosis of CMUSE. However, Crohn's disease is frequently seen in the jejunum with active illness elsewhere and jejunal involvement appears to be uncommon. One of our patients who had CMUSE had a wireless capsule endoscopy that showed a mixed inflammatory infiltrate (mainly plasma cells) on histology.

CMUSE is only one of several small intestinal diseases, each of which must be excluded when making a differential diagnosis. Also, the medications NSAIDs cause small intestinal injury, as well as tuberculosis and other chronic infections of the small bowel, Behcet's disease, drug-induced small intestinal injury (thiazides, potassium chloride) and malignancies must be ruled out. Extranodal malignant lymphoma most commonly affects the gastrointestinal system. This illness is not only found in polypoid and diffuse forms, but also in ulcerative forms. In the majority of cases, one of the following conditions is established by histology: diffuse large B-cell lymphoma or mucosa-associated lymphoid tissue (MALT) lymphoma. Rheumatoid arthritis patients experienced nonspecific ulcers in the small and large intestines while undergoing treatment with tocilizumab.

It is also required to identify CMUSE as a non-frequent pathological disease, rather than another CMUSE. We believe that CMUSE is unique from collagenous sprue, autoimmune or eosinophilic enteritis and chronic ulcerative jejunitis. Many stenosis of the small intestine is a prerequisite for diagnosing CMUSE, but nonspecific small intestinal ulcers cannot constitute CMUSE until there are multiple stenosis in the small intestine.

\section{T cell subsets:}

The cellular immunity was low, the value continued to decrease and the possibility of infectious diseases increased. Thus, it was suggested that immunopotentiators should be used appropriately on the basis of symptomatic treatment and immunopotentiators could be combined according to the patient's condition to reduce the incidence of infection. The dynamic level of cellular immunity was measured again during the medication cycle (fig. 4). 

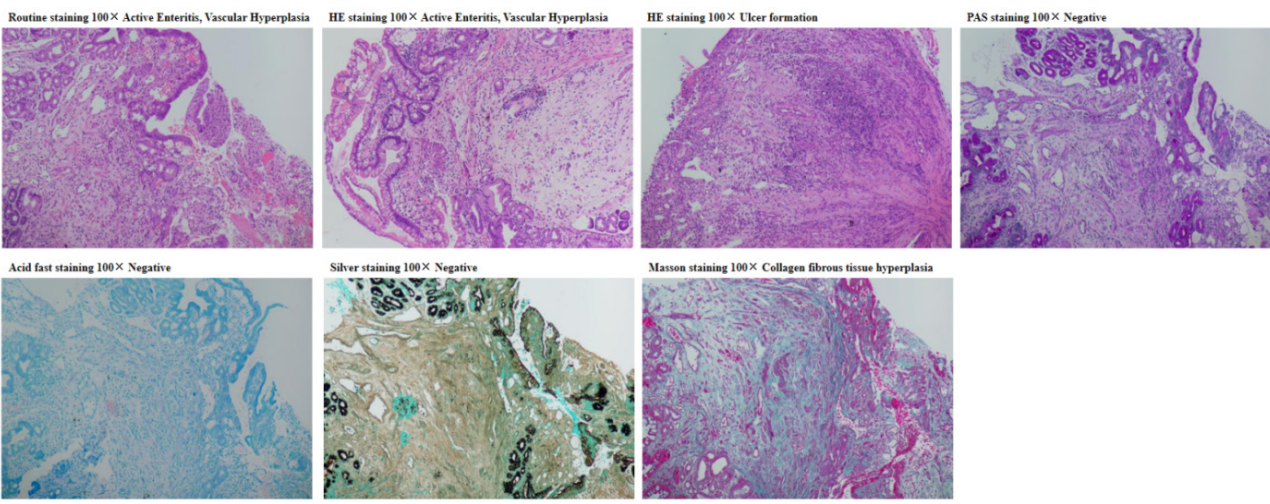

Fig. 4: Biopsy pathology of the small intestinal ulcer

Nephrotic syndrome is a clinical syndrome caused by various kidney diseases. The permeability of the glomerular basement membrane is increased. The basic characteristics include a large amount of proteinuria, that is, adults $>3.5 \mathrm{~g} / \mathrm{d}$ or random urine albumin to creatinine ratio (ACR) as the standard, such as ACR > $2200 \mathrm{mg} / \mathrm{g}$; hypoalbuminemia (plasma albumin $<30 \mathrm{~g} / \mathrm{l}$ ); edema of varying degrees is often accompanied by hyperlipidemia, of which the first two are necessary conditions for diagnosis. Pathological manifestations such as minimal changes, mesangial proliferative glomerulonephritis, mesangial capillary glomerulonephritis, membranous nephropathy, focal segmental glomerulosclerosis, etc. are common. The patient has thrombotic microangiopathy and is not a common pathological manifestation of nephrotic syndrome. Thrombotic microangiopathy is a group of acute clinicopathological syndromes, the main characteristics are microangiopathic hemolytic anemia, platelet decline and microvascular thrombosis. Acute renal failure is often caused when the kidneys are involved. Proteinuria and hematuria generally indicate kidney damage.

\section{MANAGEMENT}

At the moment, no remedy is available that can fix the problem. Systemic glucocorticosteroids should be the first line of therapy ${ }^{[16]}$. The dosage of systemic glucocorticosteroids varies inter individually; some people require prednisone ( $20 \mathrm{mg}$ daily) while others might profit with dexamethasone ( $9 \mathrm{mg}$ daily). But the majority of patients will have to endure the consequences of corticosteroid dependency ${ }^{[17]}$. Enteral or parenteral nutrition, in combination with iron supplementation, can be helpful, only until the time of delivery. Unfortunately, there was no response to treatment with 5-aminosalicylic acid and azathioprine. Patient's minor intestinal strictures were not prevented and the mucosal healing in the gut was not achieved with this treatment.

Several fibrous strictures formerly excised surgically were removed by open surgery; currently, the fibrous strictures can be treated endoscopically via deep enteroscopy. If treated with both corticosteroids and balloon dilation of small intestine non-ulcerated stenosis, discomfort and stenosis diminish (confirmed on deep enteroscopy). At the present time, due to the development of deep enteroscopy, surgery is not considered a therapy of first choice for those with small bowel syndrome (when severe ileus episode occurs and cannot be managed by the pharmacology means of treatment, that is, intravenously administrated glucocorticosteroids) $)^{[18]}$.

\section{PROGNOSIS}

CMUSE prognosis is unknown. In the past, patients who developed strictures in the small intestine were sent to surgery. The recurrence rate after surgery was higher than expected ${ }^{[19]}$. Because of recurrent stenosis and a number of resections of the small intestine, this patient was operated 7 times.

\section{DISCUSSION}

CMUSE is a rare syndrome with unknown etiology and mainly involving small intestine, which was firstly reported by Lindholmer et al. in 1964 ${ }^{[1,20]}$. CMUSE is characterized by multiple sites and segments of the small intestine with circular shallow ulcers with stenosis, often manifested as recurrent abdominal pain, intestinal obstruction and anemia in clinic. At present, the total number of reported cases in the world is less than $100^{[2-9]}$, including about 10 cases reported in China ${ }^{[13-15]}$.

CMUSE often occurs in young and middle-aged people, but it has also been reported in children. 
Because the lesion is located in the small intestine, the diagnosis usually takes a long time due to the limitation of examination conditions and understanding of the disease. Many studies report that it usually takes years to decades from the onset of the disease to the consideration of CMUSE diagnosis ${ }^{[16]}$. The patient had intussusception $1 \mathrm{y}$ ago and was treated with surgery. Because of the lack of specificity of surgical pathology, the diagnosis was not clear.

Abdominal pain, anemia and gastrointestinal bleeding were the main manifestations of the disease in the early stage, while repeated intestinal obstruction, malnutrition and hypoproteinemia appeared in the later stage. Patients with CMUSE rarely have extraintestinal manifestations, only 4 cases of oral ulcer, anal abscess, alopecia with photoallergy and edema with polyserositis, respectively, were reported before ${ }^{[14]}$. The patient reported in the present case was complicated with nephrotic syndrome and hypothyroidism. Renal biopsy confirmed that $\mathrm{IgM}^{+}$and $\mathrm{C}^{++}$were diffusely distributed and deposited in mesangial area and vascular loop. However, it is not clear whether nephrotic syndrome is a separate disease or an extraintestinal manifestation of CMUSE.

The laboratory examination of CMUSE patients lacks specificity. In addition to anemia, hypoproteinemia, fecal occult blood positive and other non-specific manifestations, systemic inflammatory indicators such as erythrocyte sedimentation rate (ESR) and C-reactive protein were normal or slightly increased, while intestinal inflammatory indicators such as fecal calprotectin could be significantly increased. Autoantibodies were negative. There were also a few patients with abnormal immune indexes. ESR and C-reactive protein were normal in this patient. Fecal calprotectin test: $1137.3 \mu \mathrm{g} / \mathrm{g}$, which was significantly increased, indicating inflammatory lesions in the intestine. As no obvious abnormality was found in the colonoscopy, the diagnosis was finally confirmed by enteroscopy. The patient's immunoglobulin was significantly decreased, which expressed as severe hypoproteinemia and hypoimmunoglobulinemia. This may be related to this disease, but the clinical consideration is more related to nephrotic syndrome.

The diagnosis of this disease depends on endoscopic findings, therefore, capsule endoscopy and enteroscopy have high diagnostic value for this disease. Under CMUSE endoscopy, multiple small intestinal strictures and superficial ulcers are found. The lesions are mainly located in jejunum and ileum. Normal mucosa can be found between the multiple ulcers. The ulcers are various in shapes, which can be round, circular, linear or irregular. The ulcers only involve the mucosa and submucosa instead of muscle layer and below, so they do not form through wall ulcer, fistula or paving stone like changes. The biopsy specimens show nonspecific mixed inflammation of small intestinal mucosa and submucosa, which often infiltrated plasma cells, monocytes (including $\mathrm{B}$ and $\mathrm{T}$ lymphocytes), neutrophils and eosinophils ${ }^{[21]}$. At present, there are no clear and unified diagnostic criteria for CMUSE. This case was based on the diagnostic criteria of CMUSE proposed by Perlemuter et al. in $2017^{[9]}$ as follows:

Clinical history- long course of disease, several years to decades; persistent occult blood in gastrointestinal tract with severe anemia; partial family history.

Clinical features-chronic recurrent intestinal obstruction; chronic iron deficiency anemia with fatigue, dizziness, edema or growth retardation; rarely with diarrhea; normal inflammatory indicators or other systemic inflammatory biological indicators; normal colonic and gastric tissues and no extraintestinal manifestations.

Imaging findings-Enema examination showed multiple continuous stenosis of small intestine.

Endoscopic findings-multiple and pleomorphic superficial ulcers with clear boundary with surrounding normal mucosa; capsule endoscopy should be cautious because it may stay in the stenosis.

Histological findings-necrotizing inflammatory ulcer did not invade the lamina propria; nonspecific inflammation, submucosal erosion with fibrosis.

Crohn's disease, NSAIDs associated enteritis, other chronic small intestinal infections, drug-induced small bowel damage and tumors should be excluded. According to the criteria, the diagnosis of this case was clear. At present, the main treatment of CMUSE is still lack of unified and effective drugs. Currently, nutritional support and steroid therapy are mainly used. Intestinal resection or balloon dilatation are often required when stenosis and intestinal obstruction occur ${ }^{[21]}$. However, steroid dependence and poor effect were found in steroid therapy before ${ }^{[22]}$. Generally speaking, steroids are effective for early lesions with obvious inflammation. Other drugs such as mesalazine, azathioprine and 
infliximab were reported to be effective as well ${ }^{[18]}$.

The prognosis of CMUSE is often poor. Patients with recurrent ulcers need surgical treatment or even repeated surgery after stenosis ${ }^{[23]}$. Severe cases may die of malnutrition and related complications ${ }^{[23,24]}$. CMUSE related tumors have not been reported yet. The patient in this case has mild stenosis and abdominal pain, so active treatment is needed to delay the recurrence and operation time and improve the quality of life.

Whether the onset of CMUSE in this patient is related to thrombotic microangiopathy is still unclear and it is more likely to consider secondary changes in this disease.

\section{CONCLUSION}

Although it's an uncommon disorder, we recommend that CMUSE (chronic or relapsing subileus episodes resulting from numerous small intestinal strictures) should be taken into consideration when chronic or recurrent subileus episodes develop from several shallow ulcers of the small intestines. Endoscopic therapy of stenosis, if effective, can avoid surgery and unnecessary small bowel resections.

\section{Acknowledgements:}

This work was supported by Jiangsu Province Hospital of Chinese Medicine.

\section{Conflicts of interest:}

The authors report no conflicts of interest.

\section{REFERENCES}

1. Lindholmer B, Nyman E, Raef L. Nonspecific stenosing ulceration of the small bowel: a preliminary report. Acta Chir Scand 1964;128:310-1.

2. Matsumoto T, Iida M, Matsui T, Yao T. Chronic nonspecific multiple ulcers of the small intestine: a proposal of the entity from Japanese gastroenterologists to Western enteroscopists. Gastrointest Endosc 2007;66(3):S99-107.

3. Kohoutova D, Bartova J, Tacheci I, Rejchrt S, Repak R, Kopacova M, et al. Cryptogenic multifocal ulcerous stenosing enteritis: a review of the literature. Gastroenterol Res Pract 2013;2013.

4. Chang DK, Kim JJ, Choi H, Eun CS, Han DS, Byeon JS, et al. Double balloon endoscopy in small intestinal Crohn's disease and other inflammatory diseases such as cryptogenic multifocal ulcerous stenosing enteritis (CMUSE). Gastrointest Endosc 2007;66(3):S96-8.

5. Kijmassuwan T, Tanpowpong P, Molagool S, Treepongkaruna $\mathrm{S}$. A rare cause of multiple small bowel ulcers and strictures in a 10-year-old child. Turk J Gastroenterol 2018 ;29(1):112-5.

6. Hwang J, Kim JS, Kim AY, Lim JS, Kim SH, Kim MJ, et al. Cryptogenic multifocal ulcerous stenosing enteritis: radiologic features and clinical behavior. World J Gastroenterol 2017;23(25):4615-23.
7. Kwon SO, Kim YS, Kim SY, Hong SW, Lee HK, Moon JS. A case of cryptogenic multifocal ulcerous stenosing enteritis: differential diagnosis from Crohn's disease. J Gastrointestin Liver Dis 2012;21(3):309-12.

8. Chang DK, Kim JJ, Choi H, Eun CS, Han DS, Byeon JS, et al. Double balloon endoscopy in small intestinal Crohn's disease and other inflammatory diseases such as cryptogenic multifocal ulcerous stenosing enteritis (CMUSE). Gastrointest Endosc 2007;66(3):S96-8.

9. Perlemuter G, Guillevin L, Legman P, Weiss L, Couturier D, Chaussade S. Cryptogenetic multifocal ulcerous stenosing enteritis: an atypical type of vasculitis or a disease mimicking vasculitis. Gut 2001;48(3):333-8.

10. Tao EW, Zou TH, Wang YF, Tang JT, Chen YX, Gao QY. Case report of cryptogenic multifocal ulcerous stenosing enteritis (CMUSE): a rare disease may contribute to endoscopycapsule retention in the small intestine. BMC Gastroenterol 2019;19(1):1-6.

11. Yang Y, Zhao L, Zhang Y. A Steroid-resistant cryptogenic multifocal ulcerous stenosing enteritis. Niger J Clin Pract 2018;21(5):678-80.

12. Fraile G, Norman F, Eugenia Reguero M, Defargues V, Redondo C. Cryptogenic multifocal ulcerous stenosing enteritis (CMUSE) in a man with a diagnosis of $\mathrm{X}$-linked reticulate pigmentary disorder (PDR). Scand J Gastroenterol 2008;43(4):506-10.

13. Zhang Y, Huang L, Liu R, Wang M, Jiang Z, Zhou W, et al. Case report of a pair of siblings with cryptogenic multifocal ulcerating stenosing enteritis: a rare disease easily to be misdiagnosed as Crohn disease. Medicine 2017;96(32):e7527.

14. Reyihanguli A, Ye M, Jiang CQ. Cryptogenic multiifocal ulcerous stenosing enteritis:a case report and literature review. Chin J Diffic Complicated Cases 2020;8:845-6.

15. Singh A, Sahu MK, Panigrahi MK, Misra D. Cryptogenic multifocal ulcerous stenosing enteritis (CMUSE): a tale of three decades. ACG Case Rep J 2017;4.

16. Zhang $\mathrm{P}$, Sun $\mathrm{H}$, Zhang $\mathrm{Y}$, Wu J, Wang YH, Jiang ZN, et al. Cryptogenic multifocal ulcerative stenotic colitis in a child: a case report and literature review. Chin J Evid Based Pediatr 2018;3:210-4.

17. Wu D, Chen D, Liu W, Zhou W, Qian J. Clinical analysis of 10 cases of cryptogenic multifocal ulcerative stenotic colitis. Chin J Dig 2017;37(2):79-83.

18. Zhe YB, Zhou W, Mao J. Two cases of cryptogenic multifocal ulcerative stenotic colitis. Chin J Gen Surg 2017;32(1):8.

19. Chung SH, Park SU, Cheon JH, Kim ER, Byeon JS, Ye BD, et al. Clinical characteristics and treatment outcomes of cryptogenic multifocal ulcerous stenosing enteritis in Korea. Dig Dis Sci 2015;60(9):2740-5.

20. Vasco PG, Rodriguez GF. Cryptogenia multifocal ulcerous stenosing enteritis: an entity on its own as a cause of abdominal pain, iron deficiency anemia and protein-losing enteropathy. Rev Clin Esp 2014;214(1):26-30.

21. Chung SH, Park SU, Cheon JH, Kim ER, Byeon JS, Ye BD, et al. Clinical characteristics and treatment outcomes of cryptogenic multifocal ulcerous stenosing enteritis in Korea. Dig Dis Sci 2015;60(9):2740-5.

22. de Schepper H, Macken E, van Marck V, Spinhoven M, Pelckmans $\mathrm{P}$, Moreels T. Infliximab induces remission in cryptogenic multifocal ulcerous stenosing enteritis: first case. World J Gastroenterol 2013;19(10):1661-4. 
23. Brooke MA, Longhurst HJ, Plagnol V, Kirkby NS, Mitchell JA, Ruschendorf F, et al. Cryptogenic multifocal ulcerating stenosing enteritis associated with homozygous deletion mutations in cytosolic phospholipase A2- $\alpha$. Gut 2014;63(1):96104.

24. Kohoutova D, Bures J, Tycova V, Bartova J, Tacheci I, Rejchrt $\mathrm{S}$, et al. Severe cryptogenic multifocal ulcerous stenosing enteritis. A report of three cases and review of the literature. Acta Med 2010;53(53):25-9.
This is an open access article distributed under the terms of the Creative Commons Attribution-NonCommercial-ShareAlike 3.0 License, which allows others to remix, tweak, and build upon the work non-commercially, as long as the author is credited and the new creations are licensed under the identical terms

This article was originally published in a special issue, "Diagnostic and Therapeutic Advances in Biomedical Research and Pharmaceutical Sciences"

Indian J Pharm Sci 2021:83(5) spl issue "16-23" 